\title{
X-RAY TIMING STUDIES OF GRS 1915+105
}

with the Indian X-ray Astronomy Experiment (IXAE)

B. PAUL, P. C. AGRAWAL, A. R. RAO, M. N. VAHIA AND

J. S. YADAV

Tata Institute of Fundamental Research

Homi Bhaba road, Mumbai (Bombay), 400 005, India

AND

T. M. K. MARAR, S. SEETHA AND K. KASTURIRANGAN

ISRO Satellite Centre, Bangalore, 560 017, India

\section{Introduction}

We have made photometric observations of the galactic superluminal jet source GRS $1915+105$ in the energy bands of $2-6$ and $6-18 \mathrm{keV}$ during 1997 June 12-29 and August 8-10. During our observations, different types of very intense, quasi-regular X-ray bursts have been observed from this source. We present here the light curves and the power density spectra of our observation of this source in its bright state.

\section{Observations}

Observations were made with the three Pointed Proportional Counters (PPC) of the Indian X-ray Astronomy Experiment (IXAE) onboard the Indian satellite IRS-P3. The PPCs, filled with argon-methane mixture at 800 torr pressure and working in the $2-18 \mathrm{keV}$ energy range, have a total area of $1200 \mathrm{~cm}^{2}$ and field of view of $2.3^{\circ} \times 2.3^{\circ}$. The satellite was launched from the Shriharikota (SHAR) range, India, on $1996 \mathrm{March} 21$ and was put into a near circular orbit of height $830 \mathrm{~km}$ and $98^{\circ}$ inclination. The pointing accuracy of the satellite is better than $0.1^{\circ}$.

Observations were made with a time resolution of $0.1 \mathrm{~s}$ or $1 \mathrm{~s}$ and more than 55000 seconds of useful source exposure was obtained.

\section{Results}

During the 1997 observations, the source was in a bright intensity state. A unique characteristic noted in the light curves is the presence of strong quasi-regular Xray bursts. Some representative light curves of 500 seconds duration obtained on different days with one of the PPCs are shown in the left column of Figure 1 . The power density spectra in the frequency range of 0.001 to $5 \mathrm{~Hz}$, obtained from the 

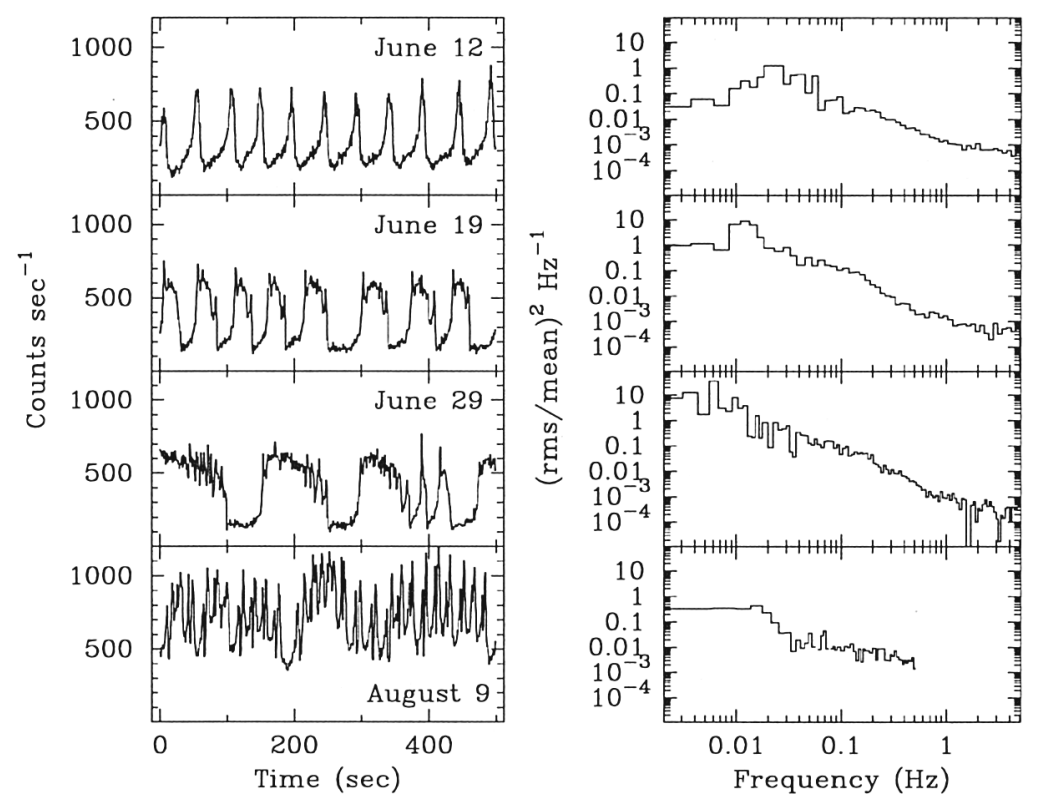

Figure 1. The light curves showing different types of bursts observed in GRS $1915+105$ are plotted in the left column along with the day of observation in 1997. The corresponding power density spectra are shown in the right column.

light curves consisting these different type of bursts are shown in the right column of the figure.

GRS $1915+105$ is the only black hole source in which regular bursts are observed. The bursts observed in this source also have the unique feature of slow rise and fast decay. The spectrum is found to be harder during the burst decay. These features make the bursts in GRS 1915+105 distinct from both the type I and type II X-ray bursts observed in neutron star sources. 